2020-10-08

\title{
Focused wave interactions with floating structures: A blind comparative study
}

\author{
Ransley, E::0000-0003-1446-7907
}

http://hdl.handle.net/10026.1/16424

10.1680/jencm.20.00006

Proceedings of the Institution of Civil Engineers: Engineering and Computational Mechanics Thomas Telford

All content in PEARL is protected by copyright law. Author manuscripts are made available in accordance with publisher policies. Please cite only the published version using the details provided on the item record or document. In the absence of an open licence (e.g. Creative Commons), permissions for further reuse of content should be sought from the publisher or author. 


\section{Focused wave interactions with floating structures: A blind comparative study}

E. J. Ransley, S. A. Brown, M. Hann and D. M. Greaves School of Engineering, Computing and Mathematics, University of Plymouth, UK

\section{Windt and J. Ringwood}

Centre for Ocean Energy Research, Maynooth University, Ireland J. Davidson

Dept. of Fluid Mechanics, Faculty of Mechanical Engineering, Budapest University of Technology and Economics, Hungary

P. Schmitt

School of Natural \& Built Environment, Queen's University Belfast, UK

S. Yan, J. X. Wang, J. H. Wang and Q. Ma

School of Mathematics, Computer Science and Engineering,

City, University of London, UK

Z. Xie

School of Engineering, Cardiff University, UK

G. Giorgi

Department of Mechanical and Aerospace Engineering,

Polytechnic University of Turin, Italy

\author{
J. Hughes, A. Williams and I. Masters \\ Zienkiewicz Centre for Computational Engineering, \\ College of Engineering, Swansea University, UK \\ Z. Lin, H. Chen, L. Qian and Z. Ma \\ Department of Computing \& Mathematics, \\ Manchester Metropolitan University, UK \\ Q. Chen, H. Ding and J. Zang \\ Dept. of Architecture \& Civil Engineering, \\ University of Bath, UK \\ J. van Rij and Y. Yu \\ National Renewable Energy Laboratory, USA \\ Z. Li, B. Bouscasse and G. Ducrozet \\ LHEEA, École Centrale de Nantes, France \\ H. Bingham \\ Department of Mechanical Engineering, \\ Technical University of Denmark, Denmark
}

The paper presents results from the Collaborative Computational Project in Wave Structure Interaction (CCP-WSI) Blind Test Series 2. Without prior access to the physical data, participants, with numerical methods ranging from low-fidelity linear models to fully nonlinear Navier-Stokes (NS) solvers, simulate the interaction between focused wave events and two separate, taut-moored, floating structures: a hemispherical-bottomed cylinder and a cylinder with a moonpool. The 'blind' numerical predictions for heave, surge, pitch and mooring load, are compared against physical measurements. Dynamic time warping is used to quantify the predictive capability of participating methods. In general, NS solvers and hybrid methods give more accurate predictions; however, heave amplitude is predicted reasonably well by all methods; and a WEC-Sim implementation, with CFD-informed viscous terms, demonstrates comparable predictive capability to even the stronger NS solvers. Large variations in the solutions are observed (even among similar methods), highlighting a need for standardisation in the numerical modelling of WSI problems.

\section{Introduction}

Numerical modelling is a crucial part of the design analysis in most engineering disciplines, particularly offshore engineering. Recently, with the continued industrialisation of the seas, engineers are readily turning to floating solutions to maximise exploitation. Consequently, there exists an overwhelming number of numerical codes to accommodate this transition and provide quantitative descriptions of the interaction of waves with floating systems. When selecting a numerical tool for a task, the model fidelity is a theoretical metric, indicating the degree of simplification adopted in the physics, as well as the corresponding mathematical model. The high-fidelity Navier-Stokes (NS) model is theoretically more accurate than lower fidelity models, e.g. the fully nonlinear potential theory (FNPT), due to its lower degree of simplification in the physics (capable of including multiphase processes as well as the effects of viscosity, turbulence and compressibility). The practical fidelity and reliability of these models, however, depends on the specific implementation, e.g. the discretisation of the governing equations, which in turn relies heavily on the experience of the operator. Further, more sophisticated methods tend to come with considerable penalties in terms of the required computational resource (prohibiting their use in routine engineering design), so typically a compromise has to be made. Therefore, although considerable overlaps in predictive capability are likely, in general, selecting an appropriate (let alone the optimal) numerical model (or implementation) is not trivial, particularly because the physical requirements of the model might not be known a priori. To complicate things further, what constitutes an acceptable result is also both case- and operator-specific, i.e. the operator might prioritise speed over accuracy or vice versa. Further still, as 


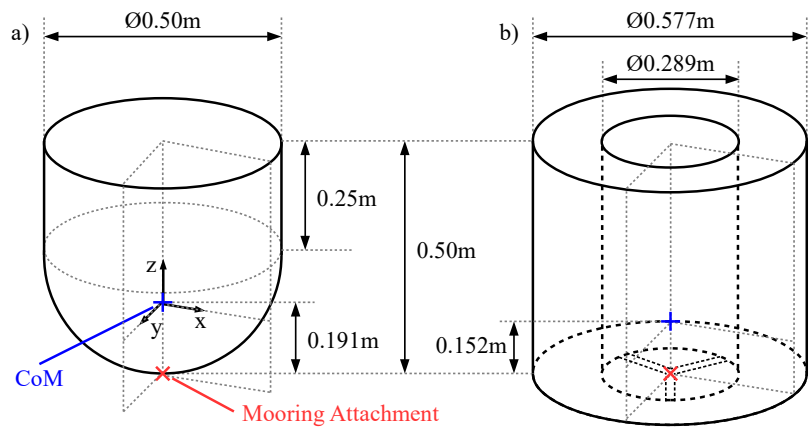

Figure 1. Dimensions, including the positions of the centre of mass (CoM) and mooring attachment, for: (a) Geometry 1 and (b) Geometry 2 [Reproduced from Brown et al. (2020a)].

discussed by Ransley et al. (2019, 2020) and as a consequence of this complexity, there is no established, or appropriate, method to quantify predictive capability. Consequently, in terms of mitigation against failures due to neglected physical phenomenon (or, conversely, against the use of excessive computing resource), developing new analysis methods to understand and quantify the predictive capability of these models is of great value to the engineering industry. This is the motivation for the CCP-WSI Blind Test Workshops.

The CCP-WSI Blind Test Workshops bring together numerical modellers from the WSI community with the goal of assessing current numerical methods and accelerating the development of numerical modelling standards. In these workshops, volunteers are invited to simulate a set of test cases, based on bespoke laboratory experiments covering a range of physical complexities, without prior access to the measured data. It is believed that, the 'blind' nature of the workshops provides a better assessment of a method's predictive capability than traditional comparative studies, in which participants can 'tune' their results to achieve the best possible match with the physical data. Further, to support contributions using all types of WSI codes, no constraints are applied to the computational implementation, and participants are encouraged to follow 'best practice' procedures. As was found in the preceding CCP-WSI Blind Test Series' (Ransley et al., 2019, 2020), however, there can be considerable differences in the implementation of a numerical model (based on operator preferences), and similar codes can display very different capabilities depending on the strategy used. This complicates the comparison, compared to studies in which a specific implementation is enforced, and these blind tests could be criticised for promoting 'competition', rather than collaboration, between participants. Despite this, these blind tests highlight a key risk to industry end users and reinforce the need for best-practice guidelines in WSI modelling as well as provide evidence of what constitutes superior implementation (that would otherwise be lost if this were constrained). They also offer additional insight that complements similar, alternative efforts (Wendt et al., 2019).

\section{CCP-WSI Blind Test Workshops - Series 2}

The CCP-WSI Blind Test Series 2 is, chronologically, the third series within the CCP-WSI Blind Test Workshops and is held in conjunction with the European Wave and Tidal Energy Conference (EWTEC). Series 2 (along with Series 3 (Ransley et al., 2020)) considers the motion response and mooring load of floating bodies and is built on the basis of the CCP-WSI Blind Test Series 1 (Ransley et al., 2019), in which the capability of participating models is compared in terms of the pressure and run-up on a fixed FPSO model. The release of the Series 2 test cases was made in September 2018, and the showcase event was held during a series of special sessions and a side event at the 13th EWTEC in Napoli, Italy (1-6 September 2019). For more information on the CCPWSI Blind Test Workshops, please visit the CCP-WSI website at http/www.ccp-wsi.ac.uk/blind_test_workshops.

\subsection{Test cases}

The CCP-WSI Blind Test Series 2 test cases consist of three focused wave events, with a range of steepness, incident upon two separate, axis-symmetry, taut-moored, floating structures: a hemispherical-bottomed cylinder (Geometry 1) and a truncated cylinder with a cylindrical moonpool (Geometry 2) (Figure 1). The two geometries are designed to resemble simplified wave energy converters (WECs). The two geometries are comparable in terms of draft and water-plane area, but Geometry 2 is considered to be, geometrically, more complex (due to the 'internal' volume of water in the moonpool). The Series 2 test cases are similar to the Series 3 test cases (Ransley et al., 2020); the two structures and the mooring line (stiffness $=67 \mathrm{~N} / \mathrm{m}$; rest length $=2.199 \mathrm{~m}$ ) are the same; the experiments were all performed in the COAST Laboratory Ocean Basin $(35 \mathrm{~m} \times 15.5 \mathrm{~m})$, with a water depth, $h$, of $3.0 \mathrm{~m}$; and the same wave probe layout, six degrees-offreedom (6DOF) motion capture system and mooring load measurement, is used. For further information, the reader is referred to Ransley et al. (2020) and the online description in the CCP-WSI Data Repository (https://www.ccp-wsi.ac.uk/data_ repository/test_cases/test_case_004). For completeness, the key parameters describing the structures and the experimental setup are give in Tables 1 and 2, respectively. Complementary work involving Geometry 1 can be found in Hann et al. (2015), Lind et al. (2016), Ransley et al. (2017) and Chen et al. (2019d). The key differences between the CCP-WSI Blind Test Series 2 test cases and the Series 3 test cases (Ransley et al., 2020) are in the incident wave properties. In Series 3, a range of incident wave steepness is achieved by varying the crest elevation of a NewWave-type focused wave event (Tromans et al., 1991) with the same underlying frequency spectrum (a PiersonMoskowitz (PM) spectrum, $H_{s}=0.274 \mathrm{~m}, f_{p}=0.4 \mathrm{~Hz}$ ). Here, a range of wave steepness is achieved by adjusting the underlying frequency spectrum whilst maintaining the same crest elevation. It is hoped that, combined, these two studies will help to differentiate between the effect of wave amplitude and frequency content on the predictive capability of the numerical models. 


\begin{tabular}{lll}
\hline & Geometry $\mathbf{1}$ & Geometry 2 \\
\hline mass $[\mathrm{kg}]$ & 43.674 & 61.459 \\
$\boldsymbol{z}_{\text {com,rel }}\left[\mathrm{kgm}^{2}\right]$ & 0.191 & 0.152 \\
$\boldsymbol{I}_{x x}=\boldsymbol{I}_{y y}\left[\mathrm{kgm}^{2}\right]$ & 1.620 & 3.560 \\
$\boldsymbol{I}_{z z}\left[\mathrm{kgm}^{2}\right]$ & 1.143 & 3.298 \\
draft $[\mathrm{m}]$ & 0.322 & 0.330 \\
mooring rest-tension $[\mathrm{N}]$ & 32.07 & 31.55 \\
$\boldsymbol{z}_{\text {com,rest }}\left[\mathrm{kgm}^{2}\right]$ & -0.131 & -0.178 \\
\hline
\end{tabular}

Table 1. Key parameters describing the structures and mooring line (positive $z$ is vertically upwards and $z=0$ corresponds to the still water level; $\boldsymbol{z}_{\mathrm{com}, \mathrm{rel}}$ is the distance from the mooring attachment to the CoM); $\boldsymbol{z}_{\text {com,rest }}$ is the $z$-position of the CoM when at rest).

\begin{tabular}{lllllll}
\hline Probe & $\mathbf{1}$ & $\mathbf{2}$ & $\mathbf{3}$ & $\mathbf{4}$ & $\mathbf{6}$ & $\mathbf{7}$ \\
\hline $\boldsymbol{x}[\mathrm{m}]$ & -1.50 & -2.00 & -2.75 & -4.25 & 1.50 & 2.00 \\
$\boldsymbol{y}[\mathrm{m}]$ & 0 & 0 & 0 & 0 & 0 & 0 \\
\hline \hline Probe & $\mathbf{8}$ & $\mathbf{9}$ & $\mathbf{1 0}$ & $\mathbf{1 1}$ & $\mathbf{1 2}$ & $\mathbf{1 3}$ \\
\hline $\boldsymbol{x}[\mathrm{m}]$ & 2.75 & -1.50 & 0 & 1.50 & 0 & 0 \\
$\boldsymbol{y}[\mathrm{m}]$ & 0 & -1.50 & -1.50 & -1.50 & -2.00 & -2.75 \\
\hline
\end{tabular}

Table 2. Wave probe positions relative to probe 5 (positioned at $(14.8,-0.278) \mathrm{m}$ relative to the wavemakers and centreline of the basin). Probe 5 corresponds to the position of the structure's CoM in cases with the structure included. $x$ is the direction of wave propagation; $y$ is defined using the right-hand rule.

\begin{tabular}{llllll}
\hline Case & $\boldsymbol{A}_{\boldsymbol{n}}[\mathrm{m}]$ & $\boldsymbol{f}_{\boldsymbol{p}}[\mathrm{Hz}]$ & $\boldsymbol{h}[\mathrm{m}]$ & $\boldsymbol{H}_{\boldsymbol{s}}[\mathrm{m}]$ & $\boldsymbol{k} \boldsymbol{A}[\mathrm{m}]$ \\
\hline 1BT2 & 0.25 & 0.3578 & 3.0 & 0.274 & 0.128778 \\
2BT2 & 0.25 & 0.4 & 3.0 & 0.274 & 0.160972 \\
3BT2 & 0.25 & 0.4382 & 3.0 & 0.274 & 0.193167 \\
\hline
\end{tabular}

Table 3. Parameters describing the wave conditions used in the CCP-WSI Blind Test Series 2 test cases.

As in Ransley et al. (2020), each wave is generated in the physical basin according to the facility's paddle control software and based on the linear superposition of 244 wave fronts with frequencies between 0.1 and $2 \mathrm{~Hz}$. All the waves are crest-focused, i.e. each of the contributing wave components has a phase of 0 at a theoretical focus location, $x_{0}$. The amplitudes of the components are derived by applying the NewWave formulation (Tromans et al., 1991) to a PM spectrum with the spectral parameters given in Table 3. Each wave front is then transformed back to the position of the wave paddles by the control software, and $x_{0}$ is iteratively adjusted (as described by Hann et al. (2015)) to pragmatically ensure 'focusing', i.e. a symmetric event, at the position coincident with the centre of the structure(s), i.e. wave gauge 5 .

\section{Participating Codes}

The CCP-WSI Blind Test Series 2 involved 30 volunteers from 13 institutions. There were 11 submissions ranging from linear potential theory (LPT) to Navier-Stokes (NS) solvers, including; hybrid (coupled) methods, partial-particle methods, finite element methods (FEM), finite difference methods (FDM) and finite volume methods (FVM); open-source, commercial and in-house codes. Each method is described and summarised in Table 4.

\begin{tabular}{lllll}
\hline Code reference & $\begin{array}{l}\text { Discret. } \\
\text { scheme }\end{array}$ & Theory & $\begin{array}{l}\text { Free-surface } \\
\text { treatment }\end{array}$ & $\begin{array}{l}\text { Turbulence } \\
\text { treatment }\end{array}$ \\
\hline $\begin{array}{l}\text { PIC } \\
\text { (in-house) }\end{array}$ & $\begin{array}{l}\text { FDM }+ \\
\text { meshless }\end{array}$ & NS & $\begin{array}{l}\text { MAC+ } \\
\text { (1-phase) }\end{array}$ & laminar \\
\hline $\begin{array}{l}\text { OpenFOAM } \\
\text { (source-term) }\end{array}$ & FVM & NS & VOF & laminar \\
\hline $\begin{array}{l}\text { Hybrid FNPT/NS } \\
\text { (in-house) }\end{array}$ & $\begin{array}{l}\text { FEM/ } \\
\text { FVM }\end{array}$ & $\begin{array}{l}\text { FNPT/ } \\
\text { NS }\end{array}$ & $\begin{array}{l}\text { 1-phase/ } \\
\text { VOF }\end{array}$ & $\begin{array}{l}\text { inviscid/ } \\
\text { laminar }\end{array}$ \\
\hline $\begin{array}{l}\text { LPT+WAMIT } \\
\text { (in-house) }\end{array}$ & BEM & LPT & linearised & inviscid \\
\hline $\begin{array}{l}\text { Hybrid } \\
\text { FNPT/SWENSE } \\
\text { (in-house) }\end{array}$ & FVM/ & FNPT/ & $\begin{array}{l}\text { 1-phase/ } \\
\text { VOF }\end{array}$ & $\begin{array}{l}\text { inviscid/lam- } \\
\text { inar }\end{array}$ \\
\hline $\begin{array}{l}\text { OpenFOAM } \\
\text { (overset) }\end{array}$ & FVM & NS & VOF & laminar \\
\hline STARCCM+ & FVM & NS & VOF & RANS (SST) \\
\hline WEC-Sim 1 & BEM & LPT & linearised & inviscid \\
\hline $\begin{array}{l}\text { Nonlinear } \\
\text { Froude-Krylov }\end{array}$ & Analytical & LPT & linearised & inviscid \\
\hline $\begin{array}{l}\text { OpenFOAM } \\
\text { (waves2Foam) }\end{array}$ & FVM & NS & VOF & laminar \\
\hline WEC-Sim 2 & BEM & LPT & linearised & inviscid \\
\hline & & & & \\
\hline
\end{tabular}

Table 4. Summary of numerical methods used by participants.

\subsection{Particle-in-cell (PIC) method (in-house)}

Using the hybrid Eulerian-Lagrangian PIC method, this model solves the incompressible NS equations for single-phase, freesurface flows. A Cartesian cut-cell-based, two-way coupling algorithm is used to incorporate fluid-solid interaction (Chen et al., 2019d). A piston-type wave paddle is used to generate the waves with the displacement, based on first-order wavemaker theory, derived by iteratively adjusting the theoretical focus location and amplitude. Wave absorption is achieved via an improved relaxation approach (Chen et al., 2019c). The computational domain is $6 \mathrm{~m}$ wide, $21 \mathrm{~m}$ long and $4 \mathrm{~m}$ tall and has $\sim 32$ million cells (edge length $0.025 \mathrm{~m}$ ) and $\sim 189$ million particles. Dynamic time-stepping is used with a Courant number, $C o$, of 0.5 ). No turbulence modelling is employed, i.e. laminar flow is assumed. Computations were performed using $160 \times 2.6 \mathrm{GHz}$ cores (Chen et al., 2019b).

\subsection{OpenFOAM using source-term}

Based on the open-source, FVM-based, OpenFOAM (v4.1), this model solves the two-phase, incompressible, Reynoldsaveraged NS (RANS) equations via volume-of-fluid (VOF) 
interface capturing (Rusche, 2002). Dynamic mesh-deformation accommodates the body motion. An impulse source method is used to generate the incident waves, with the source term determined via iterative calibration (Schmitt et al., 2019). A numerical beach implementation is used for wave absorption (Schmitt and Elsaesser, 2015). The computational domain is $7.825 \mathrm{~m}$ wide (utilising a symmetry-plane), $28.75 \mathrm{~m}$ long and $6 \mathrm{~m}$ tall ( $3 \mathrm{~m}$ of air phase) with $\sim 1$ million cells (edge lengths $1.9 \mathrm{~m}-0.015 \mathrm{~m}$ ). A fixed time step of $0.002 \mathrm{~s}$ is used. Laminar flow is assumed. $23 \times 2.4 \mathrm{GHz}$ cores were used for the computations (Windt et al., 2019).

\subsection{Hybrid FNPT/NS method (in-house)}

This hybrid model, qaleFOAM, combines the FNPT model, QALEFEM (Ma and Yan, 2006; Yan and Ma, 2006), with the VOF, NS solver from OpenFOAM, using a coupling boundary and domain decomposition method (Li et al., 2018). In the NS domain, body motion is via mesh-deformation. Wave generation and absorption takes place in the FNPT domain, using self-adaptive wavemakers. The FNPT domain is the size of the physical basin with a characteristic cell size of $0.075 \mathrm{~m}$. The NS domain is $3 \mathrm{~m}$ wide, $6 \mathrm{~m}$ long and $4.5 \mathrm{~m}$ tall and has $613 \mathrm{k}$ cells (edge lengths $0.02 \mathrm{~m}$ $0.1 \mathrm{~m})$. Dynamic time-stepping is used $(\mathrm{Co}=0.4)$. Laminar flow is assumed. Computations used $16 \times 2.4 \mathrm{GHz}$ cores (Yan et al., 2019).

\subsection{LPT + WAMIT method (in-house)}

This method computes the frequency-domain response of the body, including a linear spring for the mooring line, using linear potential flow theory (LPT) (WAMIT Inc., 2019; Bingham, 2019). A linear superposition of the Fourier coefficients, derived from the measured surface elevation signal at probe 5 in the empty tank test, is used to represent the incident waves. Only cases involving Geometry 1 were simulated using the LPT method; a high-order B-splines method, with 54 unknowns in total, represents the geometry exactly. One $2.5 \mathrm{GHz}$ core was used for the computations.

\subsection{Hybrid FNPT/SWENSE method (in-house)}

This method decomposes the wave-interaction problem into: an incident wave part, which is solved using a High-Order Spectral (HOS) wave model based on FNPT, HOS-NWT (Ducrozet et al., 2012); and a complementary part, which solves the two-phase, spectral wave explicit Navier-Stokes equations (SWENSE) using a VOF method (implemented in OpenFOAM), foamStar-SWENSE (Li et al., 2020). Communication between the two solvers is achieved using Grid2Grid (Choi et al., 2018). The entire physical tank is modelled; the wavemaker's motion is obtained using a time-reversal procedure (Ducrozet et al., 2020), which reproduces the surface elevation at the structure position, i.e. probe 5 . The complementary part is solved in a domain encompassing the buoy, made up of hemispherical and cylindrical parts (radius $=5 \mathrm{~m}$ ) with $\sim 0.6 \mathrm{M}$ cells (edge length $0.01 \mathrm{~m}-0.25 \mathrm{~m}$ ). Body motion is accommodated via mesh-deformation. A fixed time step of $0.005 \mathrm{~s}$ is used. Laminar flow is assumed. $24 \times 2.5 \mathrm{GHz}$ cores are used. Only cases involving Geometry 1 were simulated using the Hybrid FNPT/SWENSE method.

\subsection{OpenFOAM using overset meshing}

Based on OpenFOAM (v1706), this method solves the twophase, incompressible, NS equations using a VOF scheme. Overset meshing is used to accommodate body motion (Chen et al., 2019a). The incident waves are generated, based on the second-order irregular wave theory, with components derived from the given theoretical spectrum, (and absorbed) using IHFOAM (Higuera et al., 2013). The computational domain is $25 \mathrm{~m}$ long, $6 \mathrm{~m}$ wide and $4 \mathrm{~m}$ tall ( $1 \mathrm{~m}$ of air phase) and consists of 4.5 million cells (edge length $0.011875 \mathrm{~m}-0.25 \mathrm{~m}$ ). Dynamic time-stepping is used ( $C o=$ 0.35 ). Laminar flow is assumed. Computation is performed using $64 \times 1.7 \mathrm{GHz}$ cores (Lin et al., 2019).

\subsection{STARCCM+}

This method utilises the commercial software STAR-CCM+ v13.02 (Siemens, 2019) to solve an implicit, unsteady, 3D, RANS model using the Eulerian multiphase VOF method for the free surface. The SST $k-\omega$ turbulence model, with 'all $y+$ wall' treatment, is used. STAR-CCM+'s dynamic fluid body interaction overset method is applied. The mooring line is simulated with a simple linear spring coupling with no repelling force. Wave generation is achieved via an expression-based boundary condition based on 244 linearly superimposed wave frequencies derived from the theoretical wave descriptions. The full wave tank (including the beach geometry), accounting for symmetry, is modeled using $\sim 23$ million cells (edge length $0.0125 \mathrm{~m}-0.2 \mathrm{~m}$ ). A fixed time step of $2.15 \times 10^{-3} \mathrm{~s}$ (based on $C o=0.5$ ) is used. Computations are performed using between 192 and 384 cores with $2.3 \mathrm{GHz}$ (van Rij et al., 2019, 2020).

\subsection{WEC-Sim 1}

This method is based on LPT and utilises the open-source code WEC-Sim (NREL and Sandia, 2014) to simulate the rigid body time-domain responses by solving the Cummins' equation with linear hydrodynamic coefficients obtained from the frequencydomain code WAMIT (WAMIT Inc., 2019). Incident waves are specified using a linear superposition of components derived using an FFT of the empty tank data at probe 5. Viscous drag coefficients are estimated by tuning the WEC-Sim response to that using STAR-CCM+ (described in 3.7); mooring forces are included via a coupling with open-source code MoorDyn (Hall, 2015); weakly nonlinear restoring and Froude-Krylov forcing terms are calculated via integration over a discretised body surface (2592 panels for Geometry 1, 2048 panels for Geometry 2) at each $0.01 \mathrm{~s} \mathrm{time} \mathrm{step.}$ Computations used $8 \times 2.7 \mathrm{GHz}$ cores (van Rij et al., 2019, 2020).

\subsection{Nonlinear Froude-Krylov}

This model adds nonlinear kinematics and nonlinear FroudeKrylov force calculations to a LPT-based framework (Giorgi and Ringwood, 2018b,a). A linear superposition of components, derived from the surface elevation at probe 5 (in the empty tank tests) and modified using Wheeler-stretching, is used to approximate the incident waves, which are assumed to propagate linearly. No viscous drag correction is included. A fixed time step of $0.04 \mathrm{~s}$ is used. Computations were run on one $3.5 \mathrm{GHz}$ core (Giorgi, 2019). 


\subsection{OpenFOAM using waves2Foam}

Based on OpenFOAM (v5.0), this model uses a VOF scheme to solve the two-phase, incompressible, RANS equations (Rusche, 2002). Dynamic mesh-deformation accommodates the body motion. A linear superposition of wave components, derived using an FFT of the surface elevation at probe 1 in the empty tank test, is used to generate the incident waves via an expression-based boundary condition. A relaxation zone is used to absorb the waves (Jacobsen et al., 2012). The computational domain is $15.5 \mathrm{~m}$ wide, $25 \mathrm{~m}$ long and $6 \mathrm{~m}$ tall and consists of $\sim 11$ million cells (edge lengths $0.5 \mathrm{~m}-0.025 \mathrm{~m})$. Dynamic time-stepping is used $(C o=0.5)$. Laminar flow is assumed. $128 \times 2.5 \mathrm{GHz}$ cores were used for the computations (Brown et al., 2020b).

\subsection{WEC-Sim 2}

In this method, WEC-Sim (NREL and Sandia, 2014) calculates the rigid body time-domain responses by solving the Cummins' equation with linear hydrodynamic coefficients, for each geometry, obtained using the frequency-domain, panel-method, NEMOH (Babarit and Delhommeau, 2015) ( 2000 elements). Incident waves are derived from the instantaneous surface elevation (at probe 5) and impulse response functions calculated using BEMIO (NREL and Sandia, 2015). The mooring is included as a linear spring. Viscous drag coefficients are approximated using a steady-state, single-phase, NS solution (Geometry 1) (Hughes et al., 2019, 2020) or via empirical data (Geometry 2) (Hoerner, 1965). Nonlinear restoring or Froude-Krylov forces are not included. A $0.025 \mathrm{~s}$ time step is used. Computations used one $3.60 \mathrm{GHz}$ core.

\section{Results \& Discussion}

The CCP-WSI Blind Test Series 2 is a blind comparative study of numerical WSI codes; only surface elevation measurements from an empty physical tank test, along with the test case description above, were available to the participants prior to submission. This information was considered to be sufficient for all participants to reproduce the incident waves and, thus, the cases with the structures in place. The remaining physical data was not released until after all participants had submitted their final results, and it is these 'blind' results that are reported here.

\subsection{Physical Measurement Uncertainties}

As mentioned in the previous two blind tests (Ransley et al., 2019, 2020), well-executed and well-understood physical measurement data is critical if a conclusive assessment of a numerical model's predictive capability is sought. In this case, the experiments were performed as part of the same physical test campaign used for the CCP-WSI Blind Test Series 3 (Ransley et al., 2020); therefore, the same uncertainty due to random error is present $(<2 \%$ relative standard deviation in the maximum displacements over 5 repeat tests). As in Series 3, the greatest source of uncertainty is in the mass properties of the two structures; due to practical limitations in the compound pendulum 'swing test' used (Hinrichsen, 2014), there is potential for significant systematic uncertainty in the moments of inertia supplied to the participants, and, consequently, discrepancies in the prediction of rotational motion, i.e. pitch, might be anticipated. Despite this, as all participants have the same information, convergence of the numerical solutions, at least, is expected. Last, neither the mass or drag properties of the mooring line are known; participants were advised to assume the mooring system was 'ideal', i.e. massless, zero-stiffness and offers no resistance to the fluid flow. As a consequence, any dynamic behaviour or drag on the mooring, that was present in the experiments, will not be captured by the numerical models.

\subsection{Qualitative analysis}

\subsubsection{Incident waves}

Figure 2 shows the numerical solutions for the free-surface elevation at probe 5 (the focus location), along with the physical measurement, for the empty tank test 2BT2 (the intermediate steepness case). Also plotted is the variance density spectra of the results, $\psi_{\eta}(f)$, computed over an interrogation window of $35.3 \mathrm{~s}-50.3 \mathrm{~s}$. The linearised methods (LPT, WECSim $1 \& 2$ and Nonlinear Froude-Krylov) have not been included because these are assumed to have 'perfect' reproduction of the surface elevation at this position (the 'target' position).

As the participants had access to the empty tank data, and the situation is greatly simplified, convergence of the calculated surface elevation is expected (at least between similar methods). In general, the agreement between the numerical models and the experimental data is good, and there is less variation across the numerical solutions compared with the Series 3 results (Ransley et al., 2020) (although the participants are different and some of those with the least good results in Series 3 have not participated in Series 2); however, some of the results again demonstrate that there can still be significant discrepancies, even in this 'visible' case, depending on the implementation strategy. When viewed in frequency-space, the same observations can be made, however; the frequency-domain analysis appears to have filtered out the obvious discrepancies in the OpenFOAM (waves2Foam) result, which might only be highfrequency distortions, and has highlighted some curious issues underlying the OpenFOAM (source-term) result, which could be a consequence of the wave generation strategy used. In addition to this, it has been noticed that the quality of the reproduction varies with respect to the position interrogated and at other probe locations there is more spread in the results. Ignoring the linearised methods, which demonstrate significant discrepancies away from the target location (due to the nonlinear propagation of these waves not being described well by linear wave theory), the variations observed away from the target location highlight a clear issue with modern wave generation techniques; many of the methods that appear to perform the best use an iterative method to 'tune' their wave generation to achieve the desired surface elevation at the target location; however, if the result is not equally as good at other positions, can one be sure that the wave, including its underlying kinematics, has truly been reproduced as well as it appears to have been at the target location? 

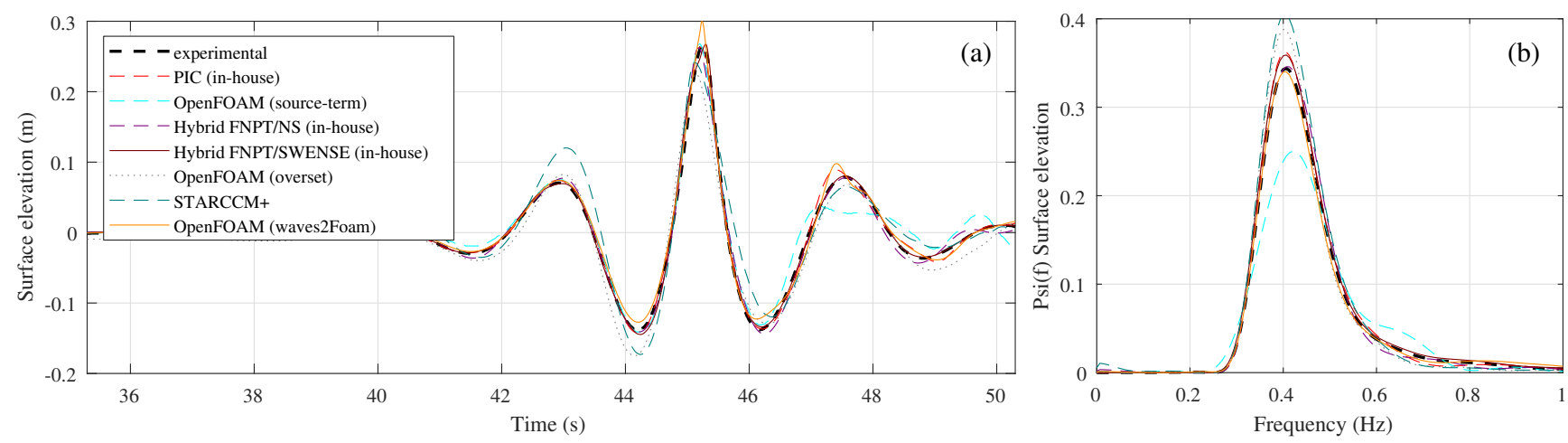

Figure 2. Surface elevation at the target position, probe 5, from the empty-tank test of the mid-steepness wave, 2BT2: (a) time series, and (b) variance density spectra (computed over an interrogation window of $35.3 \mathrm{~s}-50.3 \mathrm{~s}$ ). Physical measurements are plotted using a black dotted line; numerical submissions, from all participants, are shown using coloured lines.
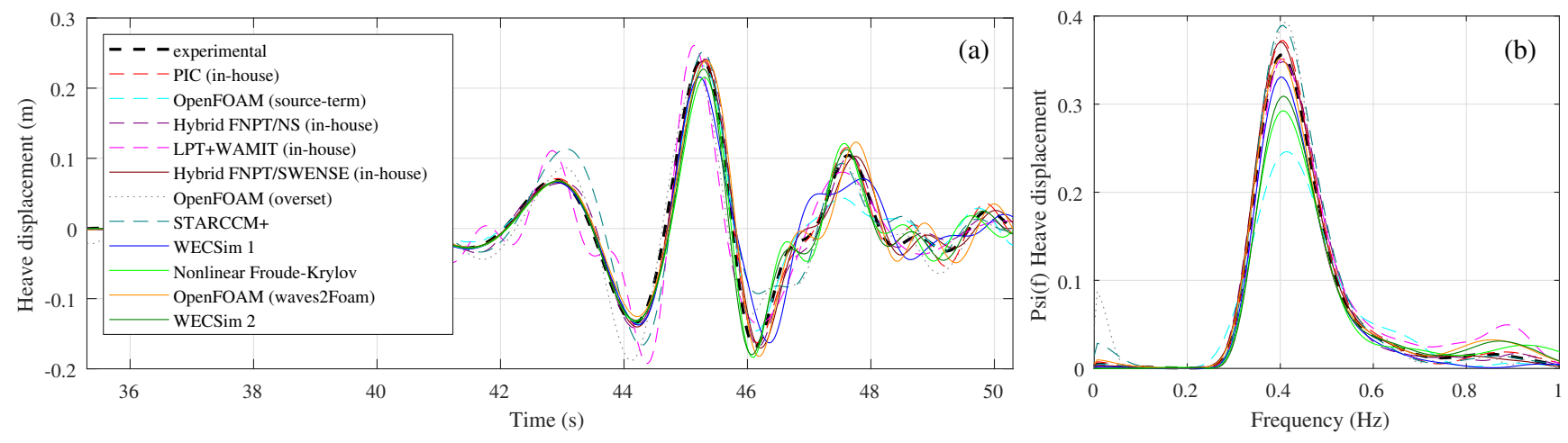

Figure 3. Heave displacement of Geometry 1's CoM when subject to the mid-steepness wave, 2BT2: (a) time series, and (b) variance density spectra (computed over an interrogation window of 35.3s - 50.3s). Physical measurements are plotted using a black dotted line; numerical submissions, from all participants, are shown using coloured lines.

Further, these methods tend to require the iterative procedure to be performed for every new case, which can increase the required computational resource considerably, particularly when using an already computational expensive method like CFD, and renders each simulation somewhat more difficult to reproduce by others. This issue is somewhat analogous to the 'transfer functions' used to control physical wavemakers which tend to be bespoke adjustments to the wavemaker displacements to achieve the desired result (typically only surface elevation is considered) at a particular position in the physical basin. This practice, in general, renders physical experiments as 'facility-specific' and, analogously, iterative wave tuning renders numerical simulations as 'method-specific' (albeit somewhat more transparent compared to the physical analogy). These observations, and the anticipated correlation between the quality of incident wave reproduction and that of the structural response, demonstrate a clear need for standardisation in numerical wave generation practices and also represent an additional complexity in judging the predictive capability of numerical models. There is no obvious trend in the quality of the reproductions as a function of wave steepness.

\subsubsection{Heave displacement}

Figure 3 shows the predicted heave displacement of Geometry 1's CoM when subject to the intermediate steepness wave, 2BT2, as well as the corresponding variance density spectra. In general the predicted heave displacement is reasonably good across all model fidelities. Possibly because, in these cases, heave motion is dominated by inertia and restoring (rather than hydrodynamic) forces; however, there is a noticeable spread in the results $( \pm 10 \%$ of max. heave) with the LPT model over-predicting and the WECSim and Froude-Krylov methods under-predicting the amplitude of heave displacement; the NS solvers typically perform slightly better, so there is some evidence to suggest the quality of the reproduction is a function of model fidelity, i.e. some nonlinear effects might be present. Despite this, as was the case in Series 3 (Ransley et al., 2020), the quality of the heave displacement prediction appears to correlate well with that of the surface elevation reproduction (for the nonlinear methods which model the wave propagation). In frequency-space, the nonlinear/propagation models again have the same trends as in the surface elevation; the LPT method has an excellent main peak but significantly 

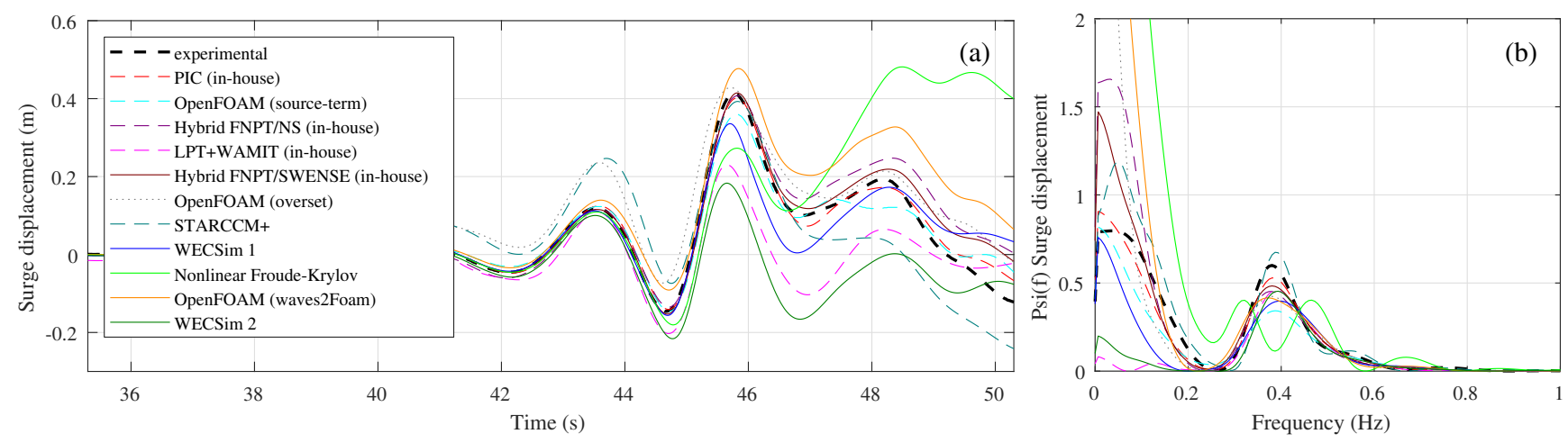

Figure 4. Surge displacement of Geometry 1's CoM when subject to the mid-steepness wave, 2BT2: (a) time series, and (b) variance density spectra (computed over an interrogation window of $35.3 \mathrm{~s}-50.3 \mathrm{~s}$ ). Physical measurements are plotted using a black dotted line; numerical submissions, from all participants, are shown using coloured lines.

overestimates the higher frequency content; the Froude-Krylov method also has noticeable disparities at high frequencies (as well as underestimating the main peak); and there is considerable variation between the two WEC-Sim results (particularly at high frequencies), perhaps suggesting that the estimate of the viscous drag and nonlinear forcing terms is important. In general, there is no obvious difference in the quality of the heave prediction with respect to the two geometries, although, for Geometry 2, the WECSim 2 implementation appears to have some issues with premature oscillation of the structure early in the time series. Again, there is no obvious trend in the quality of the reproduction as a function of wave steepness.

\subsubsection{Surge displacement}

Figure 4 shows the predicted surge displacement of Geometry 1's $\mathrm{CoM}$ when subject to the intermediate steepness wave, 2BT2, along with the corresponding variance density spectra. As in Series 3, there is considerably more spread in the surge results compared to heave, particularly after the main wave crest has passed and the structure displays significant 'drift' motion. Again, in general, the NS solvers and hybrid methods perform best and, out of these, those which reproduced the incident wave the best also appear to reproduce the surge motion the best; however, the two NS methods utilising overset meshing (STARCCM+ and OpenFOAM (overset)) do consistently predict premature surge motion that is not seen in the other methods or the physical data. Considering the linearised methods, the Froude-Krylov method does predict drift motion but has considerable discrepancies (possible due to assumed linear wave dispersion or exclusion of a viscous drag estimate); as expected, the pure LPT method does not predict any drift motion because no nonlinear effects are included; there is a clear difference between the two WEC-Sim results: WEC-Sim 2, which does not consider nonlinear forcing and has a more approximate strategy to estimate the viscous drag (Hughes et al., 2020), is missing the drift motion completely; whereas, WEC-Sim 1, which includes weakly nonlinear restoring and Froude-Krylov forcing (based on the instantaneous body position and wave elevation) and utilises a NS Solver to estimate the viscous drag coefficients (van Rij et al., 2020), gives a remarkably good prediction for the drift motion, comparable with the least good NS solvers. Despite the quality of the WEC-Sim 1 prediction, all of the linearised methods used here neglect forces from the second-order difference frequency in the spectrum (available in the NREL FAST software (Duarte et al., 2014)) as well as those from radiation and drag damping which were derived in the frequency domain by Roald et al. (2013), for example, and shown to be important in predicting the mean drift of floating structures (Stansby et al., 2019). These observations suggest that the inclusion of nonlinear forcing terms, particularly those associated with the instantaneous body position, (and, perhaps, more accurate estimates of viscous drag) are necessary to predict the surge motion in these focused wave cases. In frequency-space, the variation in the prediction of low-frequency drift motion is evident and the correlation with the incident wave prediction appears to be lost at low frequencies. Again, there is no obvious difference in the quality of the reproduction with respect to the two geometries; however, qualitatively, there is an apparent reduction in the predictive capability as a function of wave steepness (particularly for the linearised models but also generally).

\subsubsection{Pitch angle}

Figure 5 shows the predicted pitch angle of Geometry 1 when subject to the intermediate steepness wave, 2BT2, as well as the corresponding variance density spectra (results from the LPT method have been excluded due to an unresolved issue with the submitted pitch data). As was the case in the CCP-WSI Blind Test Series 3 (Ransley et al., 2020), there are considerable discrepancies in the predicted pitch motion, across all models, with a tendency, in these cases, to overestimate the amplitude and underestimate the pitch natural frequency. As mentioned earlier, the discrepancies could be a consequence of an inaccurate definition of the structures' mass properties; however, there is no convergence between the models to support this. Both the nonlinear Froude-Krylov method 

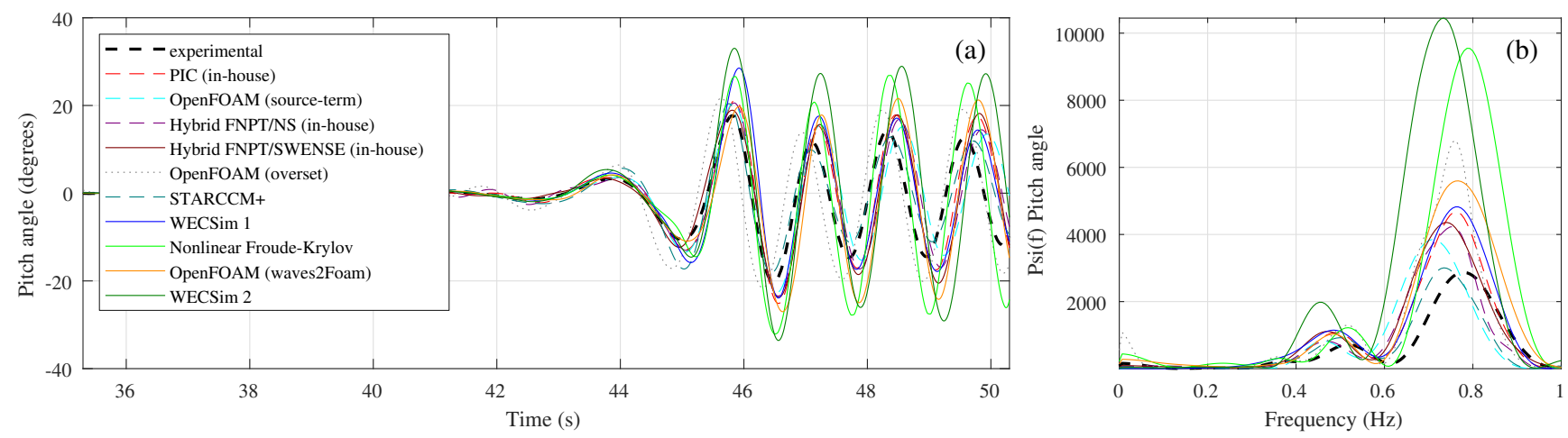

Figure 5. Pitch angle (according to the left-hand rule) of Geometry 1 when subject to the mid-steepness wave, 2BT2: (a) time series, and (b) variance density spectra (computed over an interrogation window of $35.3 \mathrm{~s}-50.3 \mathrm{~s}$ ). Physical measurements are plotted using a black dotted line; numerical submissions, from all participants, are shown using coloured lines.

and the WEC-Sim 2 contribution considerably overestimate the amplitude of pitch motion. The WEC-Sim 1 contribution also overestimates the pitch angle during the main interaction (around $46 \mathrm{~s}$ ) but then demonstrates comparable decay behaviour to the NS/hybrid solvers which, in general, perform the best (particularly close to the focus time $\sim 46 \mathrm{~s}$ ). Out of the NS solvers, it appears that the contribution utilising StarCCM+, which is the only method utilising a turbulence model $(k-\omega \mathrm{SST})$, predicts the pitch motion (of Geometry 1) most accurately, perhaps suggesting that viscous losses are important in capturing the pitch motion of floating structures (consistent with (Yan and Ma, 2007; Chen et al., 2016)). Again, there is no obvious difference in the quality of the predicted pitch motion with respect to the two geometries, except in the case of the WEC-Sim 2 and Froude-Krylov methods, which excessively overestimate the pitch amplitude of Geometry 2. This, perhaps, further exemplifies the importance of good viscous forcing estimates when predicting pitch motion, particularly when vortexshedding from edges, or sloshing effects, are more prominent. Again, there is no obvious trend in the quality of the reproductions as a function of wave steepness.

\subsubsection{Mooring load}

As in the CCP-WSI Blind Test Series 3 (Ransley et al., 2020), the mooring load in these cases is dominated by vertical motion, i.e. heave. Consequently, the quality of the reproductions is similar to that of surface elevation and heave response (with some influence from surge motion) and there is no obvious difference in the quality of the reproduction as a function of either the wave steepness or the structure's geometric complexity. Curiously, all the models predict a greater peak in the variance density of the mooring load relative to the experiment, compared to that for the heave displacement.

\subsection{Quantitative analysis}

As discussed in Ransley et al. (2019), raw time-series analysis is valuable because the data can be considered in its entirety; however, this type of analysis makes trends in the data more difficult to interpret and, typically, only qualitative assessments can be made. Frequency-domain analysis offers an alternative visualisation of the time-series data but has the same issues, when it comes to interpretation of trends. To make a quantitative analysis of the models predictive capabilities, and make progress towards a parametric understanding of the required model fidelity, the submitted data (and the physical data) need to be reduced to fewer, representative values. The challenge is, then, to identify appropriate measures of predictive capability that have no bias, towards a particular model, and are truly 'representative', i.e. independent of other factors that are considered unrelated to the 'objective function'. This can be very difficult, in general, because the objective function in question typically depends on the application (and the end user's priorities), so the most appropriate measure of predictive capability is also likely to be a function of the specific use case under investigation.

In the CCP-WSI Blind Test Series 3 (Ransley et al., 2020), a clear trend was observed between the normalised root mean squared (NRMS) error in the heave displacement and that of the surface elevation at the target location in the empty tank test. Using this measure, the capability of the models to predict heave motion was shown to correlate strongly with the quality of incident wave reproduction and the same trend is observed here (Figure 6). This suggested that all the NS solvers and hybrid methods have the same predictive capability for heave, given an equally good reproduction of the incident wave. This might have been anticipated because RMS tries to quantify the discrepancy in amplitude, and one might predict that a discrepancy in the incident wave amplitude corresponds to a discrepancy in vertical displacement, particularly when the heave is buoyancy-driven, i.e. quasi-static; however, it was noted that, although the normalisation used provides the NRMS values with some independence from the specific case and the chosen interrogation window, RMS is a fairly crude measure of 'similarity' between two time series with strong sensitivity to phase discrepancies (not just amplitude) (Ransley et al., 2019, 2020). In 


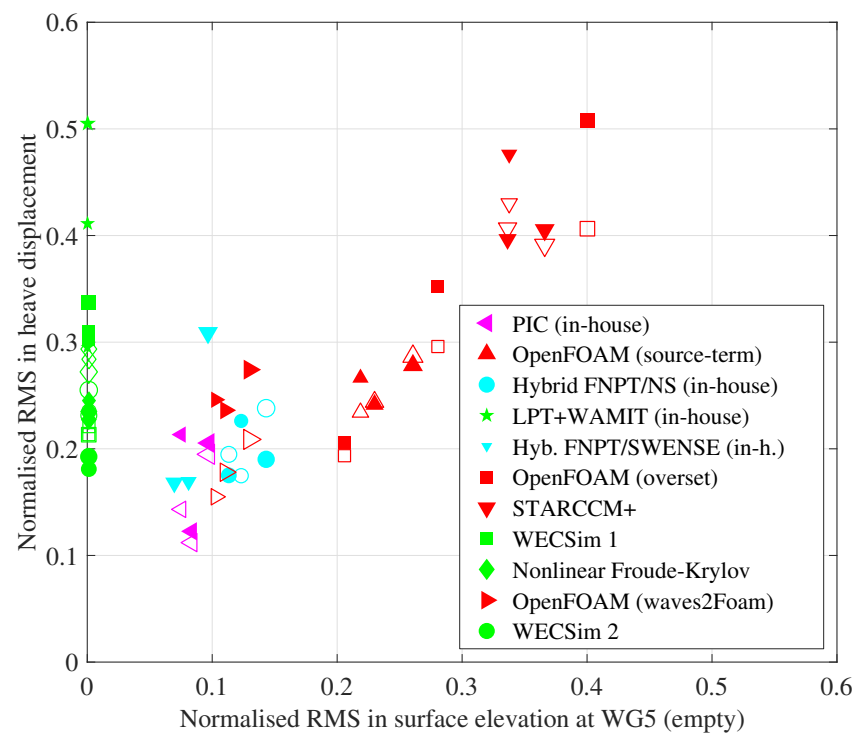

Figure 6. Normalised RMS error (w.r.t. the experimental data) in the heave displacement vs. normalised RMS error in the surface elevation at wave probe 5 (during empty tank tests). Data colour-coded by underlying theory/method (red = NS solvers, green = LPT, cyan = hybrid, magenta = PIC); filled markers $=$ Geometry 1; open markers = Geometry 2; marker sizes scaled according to the wave steepness, $k A$.

general, this makes the value of an RMS analysis questionable, because for the majority of end users, although the amplitude of a displacement might be a key objective function, it is unlikely that a small phase shift (from an otherwise perfect solution) would be reason to penalise a particular method. In the Blind Test Series 3 (and here), it appears that, despite its limitations, an RMS analysis is a reasonable assessment of predictive capability in heave (probably because no significant phase discrepancies are observed and the heave motion, in the cases considered, is dominated by buoyancy); however, it is believed that the RMS is not a representative measure of predictive capability in either surge or pitch, and this is why similar trends have not been observed in these degrees of freedom. In response to this, an alternative, novel, quantitative analysis method is proposed in an attempt to provide measures of similarity, between time series, that are independent, i.e isolate amplitude discrepancies from those in phase and frequency content.

\subsubsection{Dynamic time warping (DTW) analysis}

Dynamic time warping (DTW) is a method for measuring the similarity between two data signals that vary in time. DTW was originally designed for automatic speech recognition (Sakoe and Chiba, 1978) and, in contrast to the linear alignment of signals by cross-correlation, it is used to find the optimal global alignment between two time series by non-linearly 'warping' the time axis of the signals until their dissimilarity is minimised. This process effectively removes, from the similarity measure, the influence of both 'shifts' in the time dimension, i.e. phase discrepancies, and the 'speeds' of the two time series, i.e. frequency discrepancies, offering, what is believed to be, an independent measure of the amplitude discrepancy between two time series. In addition, a 'warping path' is generated containing the information on how to translate, compress and expand the signals so that similar features are matched. DTW has been used in many fields, from analysis of electrocardiogram (ECG) measurements (Huang and Kinsner, 2002) to fault detection in waste-water treatment (Jun, 2011) and sewer flow monitoring (Dürrenmatt et al., 2013), but, despite this, to the authors' knowledge, DTW has not been used to quantify the predictive capability of numerical models, particularly in the field of offshore and coastal engineering.

In this study, the MATLAB function, dtw, is used. Figure 7 shows an example of the results from a DTW analysis of one of the blind heave submissions (a qualitatively accurate one in this case). Compared to the original data, the 'warped' data has excellent alignment in time, with only small differences now present due only to amplitude discrepancies. Also given in Figure 7, is the minimised Euclidean distance parameter between the warped experimental and numerical data, defined as:

$$
d(\mathcal{X}, \mathcal{Y})=\sum_{w_{k}} D_{n, m}
$$

where $\mathbf{D} \in \mathbb{R}^{N \times M}$ is the distance matrix computed between all points in the two sequences, $\mathcal{X}=\left(x_{1}, x_{2}, \ldots, x_{n}, \ldots, x_{N}\right)$ and $\mathcal{Y}=$ $\left(y_{1}, y_{2}, \ldots, y_{n}, \ldots, y_{M}\right)$, given:

$$
D_{n, m}=\sqrt{\left(x_{n}-y_{m}\right)^{2}}
$$

and $W=\left(w_{1}, w_{2}, \ldots, w_{k}, \ldots, w_{K}\right)$ is the warping path, computed as a sequence of consecutive matrix elements defining a mapping between $X$ and $Y$ with the $k$-th element being $w_{k}=(n, m)_{k}$, that minimises $d$ (Dürrenmatt et al., 2013).

The warp path, for the example in Figure 7, shows noticeable compression (gradients $<1$ ), and expansion (gradients $>1$ ) is needed at the beginning and the end of the interrogation window, to align the numerical data with the experimental time series; but during the middle period the alignment is near-perfect (gradient $=$ 1). The deviations, from perfect alignment, at the beginning of the warp path might be due to low amplitudes (in heave displacement in this case), and small Euclidean distances, which can lead to unrealistic warping paths when the input signals are noisy or erroneous (Dürrenmatt et al., 2013). The deviations towards the end of the warp path might indicate the moment when inconsistencies in the reflected waves contaminate the signal. In addition, the total length and the gradients of the warp path may offer measures of similarity that are independent of discrepancies in amplitude and, therefore, an opportunity to uncover trends, similar to those observed for heave, in the prediction of other degrees of freedom like pitch and surge. 

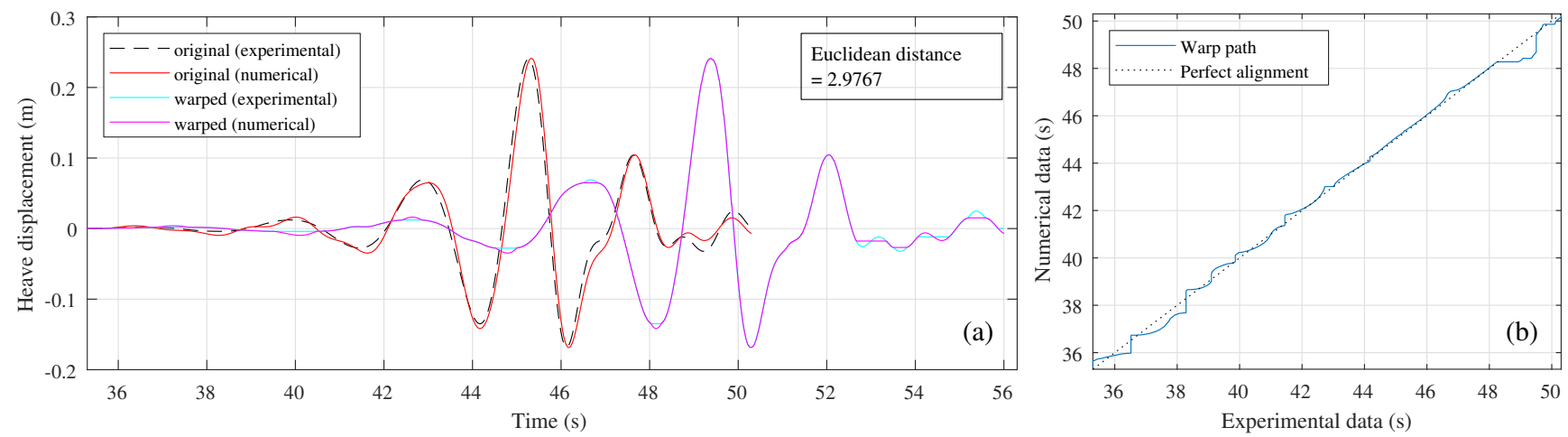

Figure 7. An example of the dynamic time warping (DTW) analysis showing: (a) the original experimental heave displacement and one of the numerical submissions, from case 2BT2 (Geometry 1), along with their warped signals and the minimised Euclidean distance between them, and; (b) the warp path compared to perfect alignment.
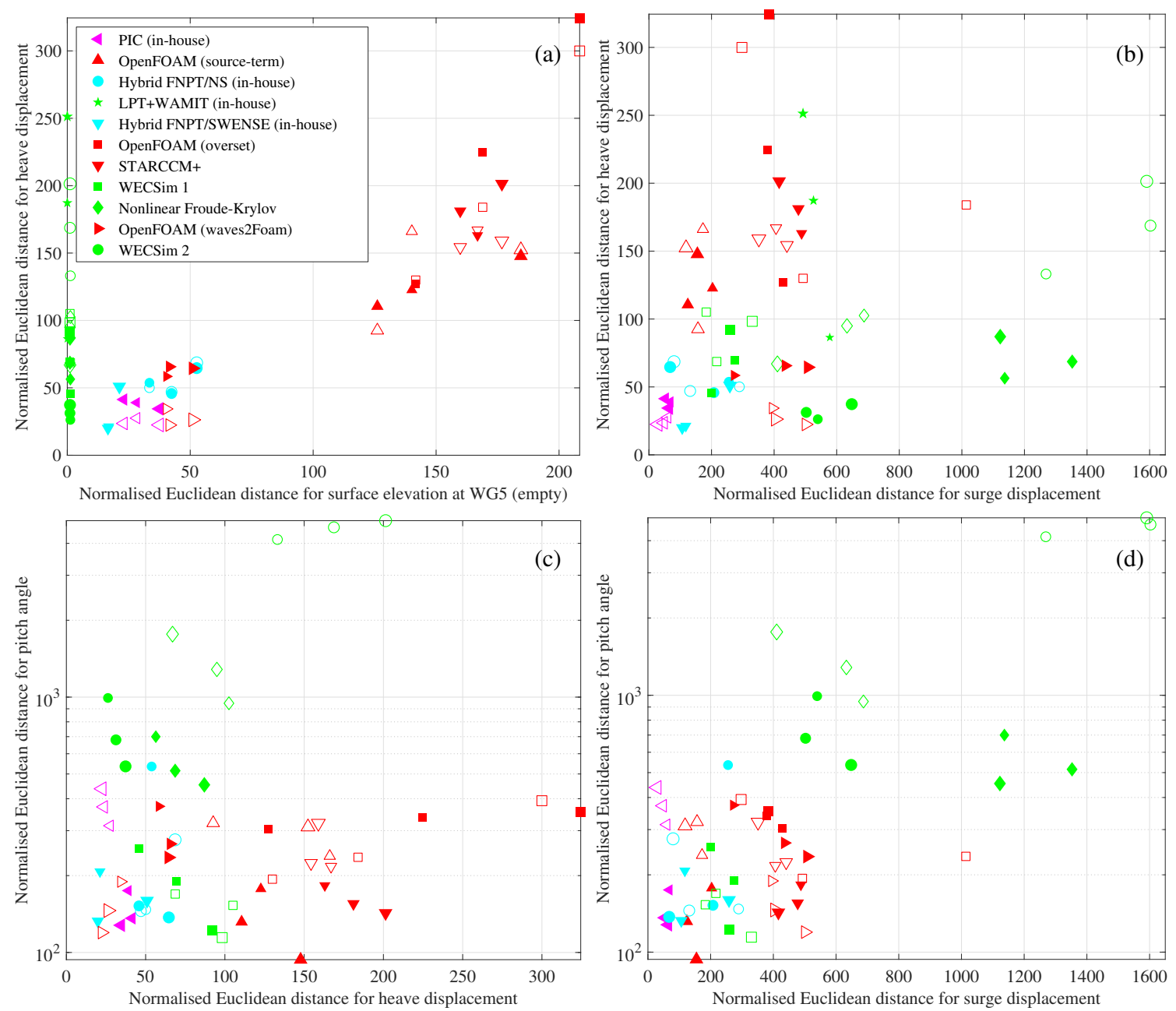

Figure 8. Normalised Euclidean distance (relative to the experimental data) for: (a) heave displacement vs. surface elevation at wave probe 5 (during empty tank tests), (b) heave vs. surge displacement, (c) pitch angle vs. heave displacement, and (d) pitch angle vs. surge displacement. Data colour-coded by underlying theory $/$ method (red = NS solvers, green $=\mathrm{LPT}$, cyan $=$ hybrid, magenta $=$ PIC); filled markers = Geometry 1; open markers = Geometry 2; marker sizes scaled according to the wave steepness, $k A$. 
Figure 8 shows the Euclidean distances between the submitted numerical data and the physical measurements. The distances are calculated over an interrogation window from $35.3 \mathrm{~s}$ to $50.3 \mathrm{~s}$, from data interpolated onto a fixed sample frequency of $128 \mathrm{~Hz}$, and are normalised by the standard deviation of the physical data over the same period (to achieve some independence with respect to the window size (Ransley et al., 2020)). Pitch results from the LPT method have been excluded due to an unresolved issue with the submitted data. Figure 8 a shows that, when using this measure, the capability of the NS/hybrid codes, to predict the amplitudes of heave motion, correlates strongly with their ability to reproduce the incident wave amplitudes. This reinforces suggestions that the heave, in these cases, is dominated by inertia and restoring (rather than hydrodynamic) forces. Compared to NRMS (Figure 6), the use of Euclidean distance appears to separate the NS/hybrid methods into two distinct groups, and the trend in the data suggests a normalised Euclidean distance of zero is achievable, for heave displacement, provided perfect reproduction of the incident wave amplitudes. This suggests that the apparent limit in the capability to predict heave motion, found using NRMS (Ransley et al., 2020), is due to phase or frequency content discrepancies and the better performing group of NS methods have relatively higher discrepancies of this type compared to the poorer performing group. Figure 8a also shows that, when using Euclidean distance (as opposed to RMS), the capability of the linearised methods to predict heave motion is apparently improved, relative to the other methods, suggesting these methods suffer more from discrepancies in frequency and phase, than amplitude, when predicting heave motion. This observation demonstrates the benefit of being able to specify the wave elevation at the position of the structure precisely because the majority of the linearised predictions of the heave amplitudes are on a par with the stronger group of NS/hybrid methods (and noticeably better than the weaker NS methods). Figure 8b shows the OpenFOAM (waves2Foam) method has a relatively poor prediction of the surge amplitudes, compared to the heave (and, therefore incident wave) amplitudes, and the OpenFOAM (source-term) method's prediction of surge amplitudes is stronger relative to heave. Other than this, in general, those NS/hybrid methods that predict the heave (and, therefore incident wave) amplitudes the best tend to predict the surge amplitudes the best (but the trend is far less pronounced compared to the heavewave relationship). For the linearised methods, the spread in the predicted surge amplitudes is large: WEC-Sim 2 and the Nonlinear Froude-Krylov method have very poor predictions for the surge amplitudes of Geometry 2 and Geometry 1, respectively; but WECSim 1 has a remarkably low Euclidean distance for surge (even noticeably lower than that of the STARCCM+ method used to tune its viscous drag coefficients). Figure $8 \mathrm{c}$ shows that the spread in the predicted pitch angle amplitudes (plotted on a log scale) is the greatest of all the degrees of freedom considered with the majority of the linearised methods giving very poor predictions, particularly for Geometry 2. The WEC-Sim 1 implementation is, again, the exception with Euclidean distances for pitch comparable to the stronger NS/hybrid methods. These observations suggest that, for cases with unbroken waves interacting with simple floating systems, lower fidelity methods, like WEC-Sim, can demonstrate predictive capabilities similar to high-fidelity NS solvers, but only if improved estimates for the nonlinear terms are found beforehand. For NS/hybrid methods, the quality of the predicted pitch amplitudes tends to follow that of the heave (and, therefore incident wave) amplitudes, but, like surge, the trend is less pronounced compared with the relationship between heave prediction and incident wave reproduction. Curiously, considering its apparently superior performance in other degrees of freedom, the PIC method has abnormally poor predictions of the pitch amplitudes for cases with Geometry 2; the OpenFOAM (sourceterm) and STARCCM+ methods also have greater pitch Euclidean distances for Geometry 2, compared to Geometry 1, but the OpenFOAM (waves2Foam) method has the opposite trend. Last, Figure $8 \mathrm{~d}$ shows the Euclidean distances for pitch versus those for surge; clearly (with the exception of WEC-SIM 1), the linearised methods are the weakest at predicting the amplitudes of pitch and surge motion; for the rest of the methods, there does not appear to be a clear trend between the Euclidean distances in pitch and surge, but, considering both degrees of freedom together, the PIC (Geometry 1), OpenFOAM (source-term) (Geometry 1) and hybrid methods (along with the WEC-Sim 1 implementation) appear superior to the rest of the NS solutions, which seem to limited by a compromise between a strong performance in either pitch or surge (but not both).

\subsubsection{Required computational resource}

As mentioned earlier, selecting the right numerical model for any particular application is considered to be a compromise between the model fidelity and the required computational effort to gain a solution. Figure 9 shows the normalised Euclidean distance for both heave and surge displacement versus the CPU effort required to generate the solutions. CPU effort has been defined as the execution time of the numerical solver (in seconds), multiplied by both the number of cores used and their speed in $\mathrm{GHz}$ then divided by the simulated time in seconds. For methods requiring 'preprocessing' steps to complete the simulations, e.g. tuning of the incident waves, the CPU effort required for these has been added when these steps must be repeated for every wave case (when the preprocessing only needs to be performed once per geometry, e.g. calculation of hydrodynamic coefficients, this effort has not been included). It should be noted that participants were not asked to minimise the CPU effort as part of this study. The WECSim 1 results have been plotted excluding the CPU effort required to estimate the viscous drag coefficients which, for the actual submitted results, required the STARCCM+ results (for each case individually) to have been found prior. This technically means the WECSim 1 CPU effort is the greatest (six orders of magnitude higher than that plotted); however, this has been considered to be misleading because typically the viscous drag coefficients for each geometry would be estimated more economically (rather than for 

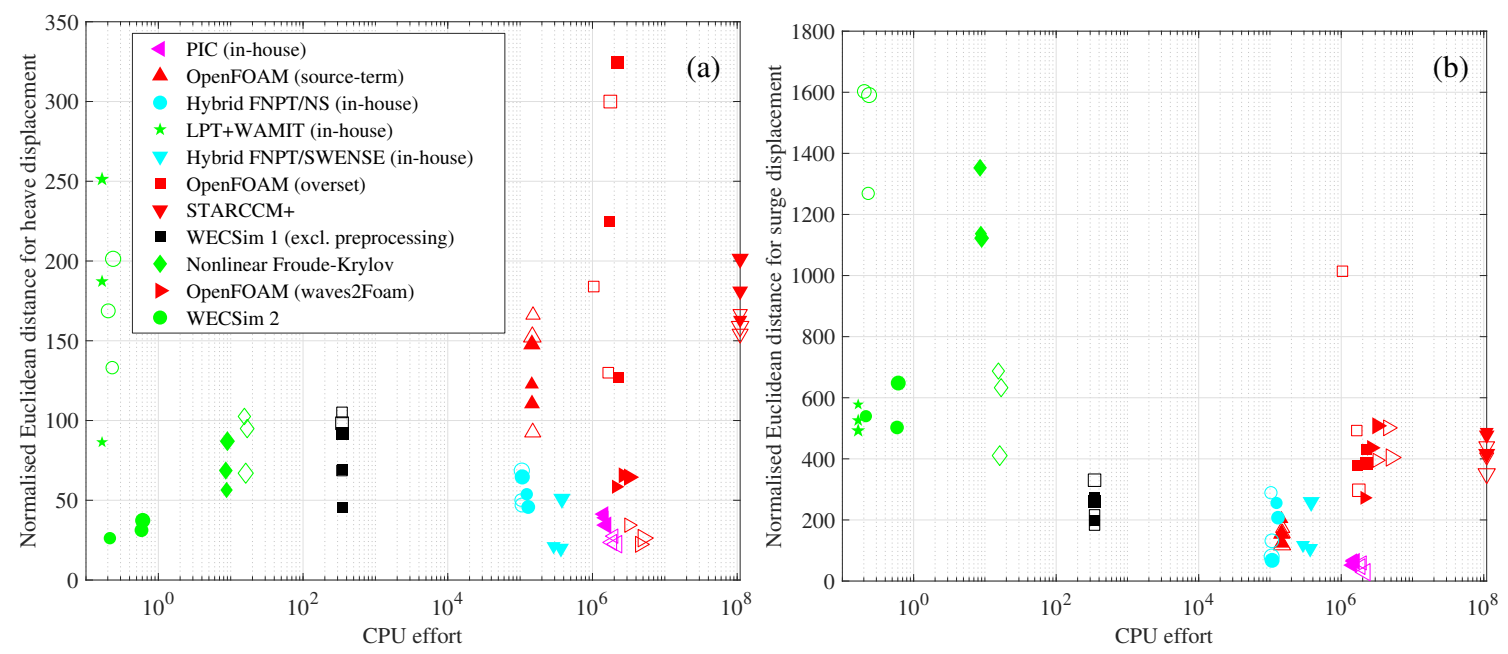

Figure 9. Normalised Euclidean distance (relative to the experimental data) for: (a) heave and (b) surge displacement vs. CPU effort. Data colour-coded by underlying theory/method (red = NS solvers, green $=$ LPT, cyan = hybrid, magenta = PIC); filled markers $=$ Geometry 1 ; open markers = Geometry 2; marker sizes scaled according to the wave steepness, $k A$. NB: WECSim 1 plotted in black as preprocessing effort has been excluded (technical CPU effort (including 'atypical' preprocessing) 10 ${ }^{8}$ ).

each incident wave case individually). Following the blind test, the use of a single set of drag coefficients (for each geometry) was found to have a fairly minimal effect on the results (van Rij et al., 2020).

As expected, the methods based on linear theory require significantly less CPU effort than the NS solvers (up to 8 orders of magnitude less) and for heave displacement, at least, there is no significant improvement in the Euclidean distance to warrant the additional computing cost (for the cases considered here). Considering the NS solvers, the two hybrid methods do demonstrate significant savings in CPU effort, with the exception of the OpenFOAM (source-term) method which exploits the symmetry of the test cases and is therefore noticeably more efficient than the other pure NS solvers. As noted in Series 1 (Ransley et al., 2019), there is a large range in the required CPU effort for the NS solvers. It is suspected that this is mainly due to the specific implementations, i.e. mesh/domain design, applied by the individual operators (rather than the efficiency of the underlying code) and, although participants were not asked to minimise the CPU effort, this highlights another opportunity for best-practice procedures to provide value.

\section{Conclusions}

The CCP-WSI Blind Test Series 2 aims to audit the numerical methods presently in use, improve our understanding with respect to the appropriate model fidelity and inform the development of future numerical modelling standards, specifically in cases involving wave interactions with floating structures. To achieve these goals, volunteers are invited to simulate a series of test cases without prior access to the measurements from equivalent physical experiments. The Series 2 test cases consist of three focused waves, with a fixed crest height but variable steepness, incident on two separate, taut-moored, floating structures: a hemisphericalbottomed cylinder (Geometry 1) and a truncated cylinder with a moonpool (Geometry 2). To maximise the number of contributions to the test, no restrictions were placed on the accepted numerical methods. Further, participants were free to chose their preferred implementation strategy, e.g. mesh design, parallel processing strategy etc. Eleven different numerical methods are used in the test, ranging from those based on LPT to NS solvers, including mesh-based and partial-particle methods, in-house, open-source and commercial codes as well as hybrid/coupled models.

Qualitative analysis of the submissions shows that, even with access to the physical data, when utilising a method that simulates the wave propagation, there can be significant differences in the quality of incident wave reproduction depending on the implementation strategy used; and the accuracy of the predicted structural response appears to correlate with that of the incident wave reproduction, particularly in the case of heave motion. In general, the NS solvers and hybrid methods do appear to have superior predictive capabilities compared to the linearised methods; however, heave is predicted reasonably well by all methods suggesting that, in these cases, the heave motion is not particularly sensitive to hydrodynamic forcing, i.e. dominated by inertia and restoring forces. For both surge and pitch, there is considerable variation in the quality of the numerical predictions (even between similar models), again highlighting the need for bestpractice guidelines in numerical implementation (Ransley et al., 
2019, 2020). In the cases considered, neither the wave steepness nor the structure's geometric complexity provide a clear distinction with respect to the required model fidelity; it is believed that the test cases, in future comparative studies, should span a step in the underlying physics, i.e. unbroken-broken wave cases, to make this distinction. In addition to this, it is paramount that the number of variables, involved in future comparative studies, be minimised by enforcing standarised implementation strategies within each numerical method. It is believed this standarisation will reduce the variability in solutions from similar methods, enable identification of subtle differences between methods solving the same equations and provide further distinction between models solving different equations; however, in order that the true predictive capability of each method is represented fairly in the comparison, the enforced implementation strategies must resemble 'best practice' for each individual method and this is presently unknown (clearly evidenced by the variability observed throughout the CCP-WSI Blind Test Workshops). To complicate things further, if one considers the common compromise between execution time and accuracy, the 'best' implementation of any particular model is also likely to be specific to the requirements of the end user.

An ancillary objective, arising from the CCP-WSI Blind Test Workshops, is the development of robust, quantitative measures of predictive capability, without which a parametric understanding of the required model fidelity might never be achieved. Here, a novel, dynamic time warping (DTW) approach is introduced in an attempt to provide such a measure. The DTW method provides a measure of similarity between two time series, the Euclidean distance, that is believed to be independent of discrepancies in both frequency and phase, making it more appropriate when quantifying amplitude discrepancies, compared to other more common analysis methods. DTW analysis also produces a separate indication of the phase and frequency discrepancies via the 'warp path' (the nonlinear warping of the time axis required to minimise the dissimilarity between the two signals being compared). The Euclidean distances between the submitted numerical data and the physical measurements provide quantitative confirmation of the qualitative observations. In general, the NS/hybrid methods perform better than the linearised methods, particularly when predicting surge and pitch motion, suggesting that at least these degrees of freedom represent a level of complexity that allows for differentiation between the predictive capabilities of different model fidelities; however, it should be noted that, there are no submissions using only FNPT so a gap is present in the model fidelities considered. As was the case in the CCP-WSI Blind Test Series 3 (Ransley et al., 2020), when simulating the cases using NS/hybrid codes, the discrepancies in heave amplitude are strongly correlated to discrepancies in incident wave amplitude; for surge and pitch amplitudes the same trend is present but is far less well pronounced. This suggests that, for those methods that simulate the wave propagation, accurate reproduction of the incident waves is absolutely paramount, if an accurate prediction of body motion is sought. Despite this, at present, there is no consensus on the best strategy to reproduce the incident waves (evident from the number of methods used in this test) and there exists large variations in the quality of those methods employed, further emphasising the need for best-practice guidelines and numerical modelling standards in WSI. For the methods based on LPT, in the cases considered here, the amplitude of heave motion is predicted reasonably well (in general, better than the weaker NS solvers); however, the amplitudes of surge and pitch motion are poorly predicted by all the LPT methods, except the WEC-Sim 1 implementation which includes both weakly nonlinear restoring and Froude-Krylov forcing as well as viscous drag terms derived using a full NS solution to the same test cases. This demonstrates that, lower fidelity methods are capable of predicting these, apparently highfidelity, degrees of freedom but, if the considerable saving in the observed computational effort is to be realised, improved methods for determining viscous drag coefficients are required. This symbolises yet another area of WSI modelling that could benefit from the development of numerical modelling standards and best-practice procedures. Finally, for the cases considered here (which, as mentioned above, all appear to represent the same physical complexity), the observations made suggest a relatively smooth trade-off between accuracy and efficiency (at least for surge and pitch motion); however, for the NS solvers the range of computational resource utilised (for effectively the same outcome) is very large and this indicates yet another area in which best practice guidelines could add value to the WSI community.

\section{Acknowledgements}

The CCP-WSI Working Group would like to acknowledge the participants of the CCP-WSI Blind Test Series 2 for their contributions as well as the European Wave and Tidal Energy Conference (EWTEC) 2019 organisers for their support. This work is funded by the Engineering and Physical Sciences Research Council (EPSRC) through projects EP/M022382/1, EP/S000747/1 and EP/P026109/1.

This work was authored in part by the National Renewable Energy Laboratory, operated by Alliance for Sustainable Energy, LLC, for the U.S. Department of Energy (DOE) under Contract No. DE-AC36-08GO28308. Funding provided by U.S. Department of Energy Office of Energy Efficiency and Renewable Energy. The views expressed in the article do not necessarily represent the views of the DOE or the U.S. Government. The U.S. Government retains and the publisher, by accepting the article for publication, acknowledges that the U.S. Government retains a nonexclusive, paid-up, irrevocable, worldwide license to publish or reproduce the published form of this work, or allow others to do so, for U.S. Government purposes.

The full physical data set from the CCP-WSI Blind Test Series 2 is available as a long standing test case for future benchmarking and can be downloaded from https://www.ccp-wsi.ac. uk/data_repository/test_cases/test_case_004. 


\section{REFERENCES}

Babarit A and Delhommeau G (2015) Theoretical and numerical aspects of the open source BEM solver NEMOH. In Proc. Of the 11th European Wave and Tidal Energy Conference, Nantes, France.

Bingham H (2019) DTUMotionSimulator package.

https://gitlab.gbar.dtu.dk/oceanwave3d/ DTUMotionsimulator.

Brown S, Musiedlak PH, Ransley E and Greaves D (2020a) Quantifying the predictive capability of OpenFOAM 5.0: Focused wave impacts with floating bodies. International Journal of Offshore and Polar Engineering 30(1): 20-27.

Brown S, Ransley E and Greaves D (2020b) Assessing focused wave impacts on floating WECs using OpenFOAM.

Proceedings of the Institution of Civil Engineers - Engineering and Computational Mechanics in press.

Chen H, Qian L, Ma Z, Bai W, Li Y, Causon D and Mingham C (2019a) Application of an overset mesh based numerical wave tank for modelling realistic free-surface hydrodynamic problems. Ocean Engineering 176: 97-117.

Chen LF, Sun L, Zang J, Hillis A and Plummer AR (2016) Numerical study of roll motion of a 2-D floating structure in viscous flow. Journal of Hydrodynamics 28: 544-563.

Chen Q, Ding H and Zang J (2019b) Application of a hybrid Eulerian Lagrangian PIC model to focused wave interaction with WEC type floating buoys. In Proceedings of the 13th European Wave and Tidal Energy Conference, Naples, Italy.

Chen Q, Kelly DM and Zang J (2019c) On the relaxation approach for wave absorption in numerical wave tanks. Ocean Engineering 187: 106210.

Chen Q, Zang J, Ning D, Blenkinsopp C and Gao J (2019d) A 3D parallel particle-in-cell solver for extreme wave interaction with floating bodies. Ocean Engineering 179: 1-12.

Choi Y, Gouin M, Ducrozet G, Bouscasse B and Ferrant P (2018) Grid2Grid : HOS wrapper program for CFD solvers. ArXiv e-prints arXiv:1801.00026.

Duarte T, Sarmento A and Jonkman J (2014) Effects of second-order hydrodynamic forces on floating offshore wind turbines. In Proceedings of AIAA SciTech, National Harbor, Maryland.

Ducrozet G, Bonnefoy F, Le Touzé D and Ferrant P (2012) A modified high-order spectral method for wavemaker modeling in a numerical wave tank. European Journal of Mechanics-B/Fluids 34: 19-34.

Ducrozet G, Bonnefoy F, Mori N, Fink M and Chabchoub A (2020) Experimental reconstruction of extreme sea waves by time reversal principle. Journal of Fluid Mechanics 884: A20.

Dürrenmatt DJ, Del Giudice D and Rieckermann J (2013) Dynamic time warping improves sewer flow monitoring. Water Research 47(11): 3803-3816.

Giorgi G (2019) CCP-WSI blind test series 2: A nonlinear Froude-Krylov modelling approach. In Proceedings of the 13th
European Wave and Tidal Energy Conference, Naples, Italy.

Giorgi G and Ringwood JV (2018a) Articulating parametric resonance for an OWC spar buoy in regular and irregular waves. Journal of Ocean Engineering and Marine Energy 4: 311-322.

Giorgi G and Ringwood JV (2018b) Relevance of pressure field accuracy for nonlinear Froude-Krylov force calculations for wave energy devices. Journal of Ocean Engineering and Marine Energy 4: 57-71.

Hall M (2015) MoorDyn User's Guide.

Hann M, Greaves D and Raby A (2015) Snatch loading of a single taut moored floating wave energy converter due to focussed wave groups. Ocean Engineering 96: 258-271.

Higuera P, Lara JL and Losada IJ (2013) Realistic wave generation and active wave absorption for Navier-Stokes models aplication to OpenFOAM ${ }^{\circledR}$. Coastal Engineering 71: 102-118.

Hinrichsen PF (2014) Bifilar suspension measurement of boat inertia parameters. Journal of Sailboat Technology Article 2014-01.

Hoerner SF (1965) Fluid-Dynamic Drag. Sighard F. Hoerner, New Jersey.

Huang B and Kinsner W (2002) ECG frame classification using dynamic time warping. In Proceedings of the Canadian Conference on Electrical and Computer Engineering, vol. 2, Winnipeg, Manitoba, Canada, pp. 1105-1110.

Hughes J, Williams A and Masters I (2019) CCP-WSI blind test series 2: Modelling focused wave interactions with floating structures with WEC-SIM. In Proceedings of the 13th European Wave and Tidal Energy Conference, Naples, Italy.

Hughes J, Williams A and Masters I (2020) Transient potential flow model of floats in focused waves compared with blind experiments. Proceedings of the Institution of Civil Engineers Engineering and Computational Mechanics (in press).

Jacobsen NG, Fuhrman DR and Fredsøe J (2012) A wave generation toolbox for the open-source CFD library: OpenFOAM ${ }^{\circledR}$. International Journal for Numerical Methods in Fluids 70: 1073-1088.

Jun BH (2011) Fault detection using dynamic time warping (dtw) algorithm and discriminant analysis for swine wastewater treatment. Journal of Hazardous Materials 185(1): 262-268.

Li Q, Yan S, Wang J and Ma Q (2018) Numerical simulation of focusing wave interaction with FPSO-like structure using FNPT-NS solver. In Proceedings of the 28th International Ocean and Polar Engineering Conference, Sapporo, Japan, pp. 1458-1464.

Li Z, Bouscasse B, Ducrozet G, Gentaz L, Le Touzé D and Ferrant P (2020) Spectral wave explicit navier-stokes equations for wave-structure interactions using two-phase computational fluid dynamics solvers. Journal of Computational Physics (in review).

Lin Z, Qian L, Ma Z, Chen H, Causon D and Mingham C (2019) Simulation of focused wave impact on point absorber wave 
energy converters - CCP-WSI blind test series 2. In Proceedings of the 13th European Wave and Tidal Energy Conference, Naples, Italy.

Lind SJ, Stansby P and Rogers BD (2016) Fixed and moored bodies in steep and breaking waves using SPH with the Froude-Krylov approximation. Journal of Ocean Engineering and Marine Energy 2: 331-354.

Ma Q and Yan S (2006) Quasi ALE finite element method for nonlinear waves. Journal of Computational Physics 212: 52-72.

NREL and Sandia (2014) WEC-Sim (Wave Energy

ConverterSIMulator). online,

https://wec-sim.github.io/WEC-Sim/ (accessed 4th February 2020).

NREL and Sandia (2015) Boundary element method input/output (bemio) documentation and users guide. online,

https://wec-sim.github.io/bemio/ (accessed 4th February 2020).

Ransley E, Greaves D, Raby A, Simmonds D and Hann M (2017) Survivability of wave energy converters using CFD. Renewable Energy 109: 235-247.

Ransley E, Yan S, Brown S, Hann M, Graham D, Windt C, Schmitt P, Davidson J, Ringwood J, Musiedlak PH, Wang J, Wang J, Ma Q, Xie Z, Zhang N, Zheng X, Giorgi G, Chen H, Lin Z, Qian L, Ma Z, Bai W, Chen Q, Zang J, Ding H, Cheng L, Zheng J, Gu H, Gong X, Liu Z, Zhuang Y, Wan D, Bingham $\mathrm{H}$ and Greaves D (2020) A blind comparative study of focused wave interactions with floating structures (CCP-WSI Blind Test Series 3). International Journal of Offshore and Polar Engineering 30(1): $1-10$.

Ransley E, Yan S, Brown S, Mai T, Graham D, Ma Q, Musiedlak PH, Engsig-Karup A, Eskilsson C, Li Q, Wang J, Xie Z, Sriram V, Stoesser T, Zhuang Y, Li Q, Wan D, Chen G, Chen H, Qian L, Ma Z, Mingham C, Causon D, Gatin I, Jasak H, Vukevi V, Downie S, Higuera P, Buldakov E, Stagonas D, Chen Q, Zang J and Greaves D (2019) A blind comparative study of focused wave interactions with a fixed FPSO-like structure (CCP-WSI Blind Test Series 1). International Journal of Offshore and Polar Engineering 29(2): 113-127.

Roald L, Jonkman J, Robertson A and Chokani N (2013) The effect of second-order hydrodynamics on floating offshore wind turbine. In Proceedings of 10th Deep Sea Offshore Wind $R \& D$ Conference, Trondheim, Norway.

Rusche H (2002) Computational fluid dynamics of dispersed two-phase flows at high phase fractions. $\mathrm{PhD}$ thesis, Imperial College of Science, Technology \& Medicine.

Sakoe H and Chiba S (1978) Dynamic-programming algorithm optimization for spoken word recognition. IEEE Transactions on Acoustics Speech and Signal Processing 26(1): 43-49.

Schmitt P and Elsaesser B (2015) A review of wave makers for 3D numerical simulations. In MARINE 2015 - Computational Methods in Marine Engineering VI, Rome, Italy, pp. 437-446.
Schmitt P, Windt C, Davidson J and Ringwood J (2019) The efficient application of an impulse source wave maker to CFD simulations. Journal of Marine Science and Engineering 7: 71.

Siemens (2019) Simcenter STAR-CCM+. online,

https://www.plm.automation.siemens.com/ global/en/products/simcenter/STAR-CCM.html (accessed 4th February 2020).

Stansby PK, Carpintero Moreno E, Apsley DD and Stallard TJ (2019) Slack-moored semi-submersible wind floater with damping plates in waves: Linear diffraction modelling with mean forces and experiments. Journal of Fluids and Structures 90: $410-431$.

Tromans PS, Anaturk AR and Hagemeijer P (1991) A new model for the kinematics of large ocean waves - application as a design wave. In Proceedings of the 1st International Offshore and Polar Engineering Conference, Edinburgh, UK, pp. 64-71.

van Rij J, Yu Y and Thanh TT (2020) Validation of simulated wave energy converter responses to focused waves. Proceedings of the Institution of Civil Engineers - Engineering and Computational Mechanics (in press).

van Rij J, Yu Y and Tom N (2019) Validation of simulated wave energy converter responses to focused waves for CCP-WSI blind test series 2. In Proceedings of the 13th European Wave and Tidal Energy Conference, Naples, Italy.

WAMIT Inc. (2019) WAMIT User Manual Version 7.3. Wamit Inc. Wendt F, Nielsen K, Yu YH, Bingham H, Eskilsson C, Kramer M, Babarit A, Bunnik T, Costello R, Crowley S, Gendron B, Giorgi G, Giorgi S, Girardin S, Greaves D, Heras P, Hoffman J, Islam $\mathrm{H}$, Jakobsen KR, Janson CE, Jansson J, Kim H, Kim JS, Kim KH, Kurniawan A, Leoni M, Mathai T, Nam BW, Park S, Rajagopalan K, Ransley E, Read R, Ringwood J, Rodrigues J, Rosenthal B, Roy A, Ruehl K, Schoeld P, Sheng W, Shiri A, Thomas S, Touzon I and Yasutaka I (2019) Ocean energy systems wave energy modelling task: Modelling, verification and validation of wave energy converters. Journal of Marine Science and Engineering 7(11): 379.

Windt C, Davidson J, Schmitt P and Ringwood J (2019) Contribution to the CCP-WSI Blind Test Series 2: CFD-based numerical wave tank experiments employing an impulse source wave maker. In Proceedings of the 13th European Wave and Tidal Energy Conference, Naples, Italy, p. 10.

Yan S and Ma Q (2006) QALEFEM for modelling 3D overturning waves. International Journal of Numerical Methods in Fluids 63: 743-768.

Yan S and Ma Q (2007) Numerical simulation of fully non-linear interaction between steep waves and 2D floating bodies using the QALE-FEM method. Journal of Computational Physics 221: 666-692.

Yan S, Wang JX, Ma QW, Wang JH and Xie ZH (2019) Numerical simulation of fucused wave interaction with WECs using a hybrid FNPT/NS solver. In Proceedings of the 13th European Wave and Tidal Energy Conference, Naples, Italy, p. 10. 